\title{
Actitudes hacia la pandemia ocasionada por el coronavirus SARS-COV-2 y la COVID-19
}

\author{
Javier Carreón-Guillén¹, José Marcos Bustos-Aguayo², Gilberto Bermúdez-Ruíz , Francisco Espinoza-Morales, \\ Cruz García-Lirios ${ }^{5}$
}

${ }^{1}$ Universidad Nacional Autónoma de México, Escuela Nacional de Trabajo Social

${ }^{2}$ Universidad Nacional Autónoma de México, Facultad de Estudios Superiores Zaragoza

${ }^{3}$ Universidad Anahuac, campus Coyoacán

${ }^{4}$ Universidad de Sonora, campus Navojoa

${ }^{5}$ Universidad Autónoma del Estado de México, Unidad Académica Profesional Huehuetoca

\section{Resumen}

El objetivo del presente trabajo fue especificar un modelo para el estudio de las actitudes hacia los efectos de la pandemia sobre el medio ambiente. Se realizó un trabajo exploratorio, transversal y correlacional con 100 estudiantes, considerando su confinamiento y uso intensivo de tecnologías, dispositivos y redes electrónicas. Se encontró una estructura factorial de tres componentes; afectivo, cognitivo e intencional con siete indicadores cada uno sumando un total de 21 la cual explicó el 35\% de la varianza total, aunque el diseño de la investigación limitó los resultados al escenario del estudio, sugiriendo la extensión del trabajo hacia la contrastación del modelo propuesto.

Palabras clave: Información, teoría, modelo, actitud, comportamiento

\section{Attitudes towards the pandemic caused by the SARS-COV-2 and COVID-19 coronavirus}

\begin{abstract}
Abstract - The objective of the present work was to specify a model for the study of attitudes towards the effects of the pandemic on the environment. An exploratory, cross-sectional and correlational work was carried out with 100 students, considering their confinement and intensive use of electronic technologies, devices and networks. A factorial structure was found that explained $35 \%$ of the total variance, although the research design limited the results to the study setting, suggesting the extension of the work towards the contrast of the proposed model.
\end{abstract}

Keywords: Information, theory, model, attitude, behavior. 


\section{INTRODUCCIÓN}

Hasta el momento en que se escribe este documento, la pandemia ocasionada por el coronavirus SARS-COV-2 y la enfermedad COVID-19 han infectado a 10 millones de personas, enfermado a cinco millones y matado a medio millón. En México, se han infectado 200 mil, han enfermado 50 mil y han muerto 25 mil (OMS, 2020). La crisis sanitaria ha obligado al confinamiento y derivado paulatinamente en una recesión y crisis económica plasmada en desempleo e inflación.

Ante este panorama desolador, las personas han desarrollado actitudes o disposiciones en contra y a favor de la situación, el gobierno, sus empleos, sus familias y ellos mismos ante la crisis sanitaria y económica, destacando las emociones de zozobra, enojo o miedo, aunque también emergen disposiciones de llevar a cabo acciones que mitiguen los efectos de la pandemia, así como estrategias de emprendimiento y colaboración solidaria y fraterna ante sus semejantes (Bustos, 2020).

De este modo, los corpus teóricos y conceptuales que explican la aparición de actitudes frente a eventos de riesgo como las pandemias sugieren; 1) emociones antropocéntricas y ecocénticas ante los recursos sanitarios; 2) evaluaciones negativas y positivas en torno a la crisis sanitaria; 3) intenciones materialistas y posmaterialistas con respecto al uso de servicios sanitarios (Hernández, 2020).

De este modo, los hallazgos más recientes y especializados advierten: a) emociones antropocéntricas asociadas al uso intensivo del agua y el papel sanitario, así como emociones ecocéntricas vinculadas a la protección de especies visitantes de las urbes; b) evaluaciones negativas de la gestión sanitaria en cuanto a escasez de pruebas, tratamientos y vacunas, así como positivas respecto al confinamiento, distanciamiento y uso de cubrebocas; c) probabilidades de eludir el autocuidado en grupos hacinados, pero seguir estas recomendaciones sanitarias en grupos pequeños (Anguiano, 2020).

La validez y fiabilidad de las respuestas al instrumento que mide la cuestión advierten; i) una consistencia interna que oscila entre 0.67 y 0.73 la cual sugiere la exclusión de reactivos para incrementarla; ii) la composición factorial exploratoria de una estructura explicada entre el 32\% y el $46 \%$ de la varianza advirtiendo la inclusión de otras dimensiones; iii) las relaciones entre factores que oscilan entre el 0.34 y 0.62 indicando la influencia de otros factores mediadores como las percepciones de riesgo y motivos de costo y beneficio ante la pandemia (Carreón, 2020).
Precisamente, el objetivo del presente trabajo es especificar un modelo para el estudio de las actitudes hacia la pandemia, los gobernantes y los gobernados, considerando los efectos de la crisis sanitaria y económica durante un confinamiento prolongado que ha durado cuatro meses y se esperan otros dos meses más con la expectativa de un nuevo rebrote de contagios, enfermedades y muertes.

La hipótesis que guía el presente trabajo sugiere una consistencia interna superior a la mínima indispensable de 0.60, una composición factorial exploratoria de tres dimensiones; afectiva, cognitiva e intencional, así como relaciones positivas y significativas entre variables latentes como manifiestas las cuales reflejan las disposiciones hacia la pandemia en estudiantes de una universidad pública del centro de México.

Los aportes de este trabajo al estado de la cuestión son; revisión del marco teórico conceptual y empírico, abordaje metodológico del problema, diagnóstico del fenómeno, discusión de los hallazgos y reflexión de aportes e implicaciones.

\section{MÉTODO}

Diseño. Se realizó un trabajo exploratorio, transversal y correlacional con una muestra de 100 estudiantes $(M=$ $24,31 D E=3,21 ; M=9^{\prime} 975,32 D E=743,23$ ingreso mensual) de una universidad pública del centro de México, considerando el confinamiento prolongado, así como el uso intensivo de tecnologías, dispositivos y redes electrónicas.

Instrumento. Se construyó la Escala de Actitudes hacia la Pandemia EAP-21) la cual incluye 21 aseveraciones en torno a tres dimensiones preponderantes; emocional-afectivasentimental ("El coronavirus afecta a los consumistas"), cognitiva ("La pandemia es un efecto del cambio climático") e intencional-conductual ("Ecologistas trabajan más en este confinamiento"). Todos los ítems se responden con alguna de cinco opciones que van desde $0=$ "nada probable" hasta $5=$ "bastante probable".

Procedimiento. Se realizó una selección intencional de 100 estudiantes de una universidad pública, considerando la clasificación de semáforos (rojo = riesgo extremo, naranja $=$ alto riesgo, amarillo $=$ riesgo moderado y verde $=$ riesgo manejable) para la observación de la pandemia en el Estado de México. Se enviaron las invitaciones a los correos institucionales de los encuestados. Una vez recibidos los acuses, se procedió a enviar el consentimiento informado, destacando el no pago por responder al cuestionario, así como la garantía de confidencialidad y anonimato tanto en la codificación como en la interpretación de las respuestas. 
Análisis. Los datos se procesaron en el paquete de análisis estadístico para ciencias sociales (SPSS por su acrónimo en inglés versión 23,0 ) considerando los requerimientos de distribución normal, fiabilidad, adecuación, esfericidad, validez, linealidad, normalidad e independencia para las estructuras de trayectorias y relaciones entre las variables a fin de poder contrastar la hipótesis nula de diferencias significativas entre las estructuras reportadas en la literatura con respecto a las observaciones realizadas en el presente trabajo.

\section{RESULTADOS}

La Tabla 1 muestra los valores de normalidad, fiabilidad y validez entre las variables que reflejan las respuestas a los reactivos que miden las actitudes hacia la pandemia, el confinamiento y la acción gubernamental como civil ante las crisis sanitaria y económica.

Establecida la estructura factorial exploratoria de los tres factores preponderantes en relación con los 21 indicadores, se procedió a estimar las relaciones entre estas tres dimensiones relativas a las actitudes emocionales, cognitivas e intencionales las cuales explicaron el 35\% de la varianza total (véase Tabla 2).

La estructura de relaciones pondera la inclusión de otro factor que la literatura identifica como ambivalencia disposicional para dar cuenta de aquellas actitudes en contra y a favor de un objeto el cual puede ser el caso de la pandemia. Es decir, por una parte, se edifican disposiciones negativas ante la crisis sanitaria y económica, pero también se abren oportunidades de trabajo inusitadas como la mensajería. De esta manera, se procedió a observar la posible emergencia de ese cuarto factor, aunque el ajuste de los tres factores también sería una opción para incrementar el porcentaje de varianza explicada (véase Figura 1).

Los parámetros de ajuste y residual $\lceil\chi 2=234.13$ (24 gl) $\mathrm{p}$ $>$.05; $\mathrm{CFI}=.990 ; \mathrm{GFI}=.999 ; \mathrm{RMSEA}=.008 \mathrm{~J}$ sugieren el no rechazo de la hipótesis nula relativa a las diferencias significativas entre la estructura de trayectorias reflejantes sustraída de la literatura con respecto a la estructura de relaciones observada.

\section{DISCUSIÓN}

El aporte del presente trabajo al estado de la cuestión radica en el establecimiento de la fiabilidad y la validez de un instrumento que mide las actitudes hacia los efectos de la pandemia en el medio ambiente, sugiriendo la extensión del trabajo hacia la contrastación del modelo en otros escenarios.
TABLA 1. Descriptivos del instrumento

\begin{tabular}{|c|c|c|c|c|c|c|c|}
\hline : & 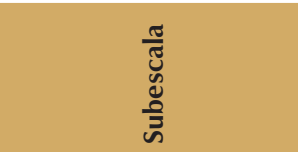 & $\frac{\pi}{\frac{\pi}{2}}$ & 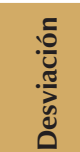 & $\stackrel{\widetilde{T}}{\gtrless}$ & 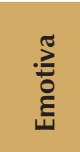 & 吝 &  \\
\hline & Emotiva & & & .781 & & & \\
\hline r1 & $\begin{array}{l}\text { El coronavirus afecta a } \\
\text { los consumistas }\end{array}$ & 4.32 & 1.34 & .762 & .365 & & \\
\hline r2 & $\begin{array}{l}\text { La pandemia sustrae } \\
\text { el ecologismo de las } \\
\text { personas }\end{array}$ & 4,15 & 1.45 & .703 & .397 & & \\
\hline r3 & $\begin{array}{l}\text { El confinamiento es } \\
\text { una solución a la } \\
\text { contaminación }\end{array}$ & 4.67 & 1.09 & .771 & .380 & & \\
\hline$r 4$ & $\begin{array}{l}\text { El uso de mascarillas es } \\
\text { para los ambientalistas }\end{array}$ & 4.83 & 1.78 & .751 & .365 & & \\
\hline r5 & $\begin{array}{l}\text { Asintomáticos también } \\
\text { contaminan }\end{array}$ & 4.05 & 1.56 & .743 & .387 & & \\
\hline r6 & $\begin{array}{l}\text { Infectados también } \\
\text { contaminan }\end{array}$ & 4.32 & 1.34 & .702 & .370 & & \\
\hline r7 & $\begin{array}{l}\text { Romper la cuarentena } \\
\text { es renunciar a la } \\
\text { movilidad } \\
\text { Cognitiva }\end{array}$ & 4.34 & 1.21 & $\begin{array}{l}.713 \\
.798\end{array}$ & .365 & & \\
\hline r8 & $\begin{array}{l}\text { La pandemia es un } \\
\text { efecto del cambio } \\
\text { climático }\end{array}$ & 4.76 & 1.01 & .743 & & .317 & \\
\hline r9 & $\begin{array}{l}\text { Animales y plantas } \\
\text { viven con coronavirus }\end{array}$ & 4.93 & 1.54 & .715 & & .396 & \\
\hline r10 & $\begin{array}{l}\text { El coronavirus hizo } \\
\text { posible la reducción de } \\
\text { emisiones }\end{array}$ & 4.36 & 1.34 & .703 & & .380 & \\
\hline r11 & $\begin{array}{l}\text { El confinamiento } \\
\text { es coexistir con las } \\
\text { especies }\end{array}$ & 4.16 & 1.55 & .762 & & .385 & \\
\hline r12 & $\begin{array}{l}\text { La cuarentena también } \\
\text { afecta a las especies }\end{array}$ & 4.30 & 1.76 & .781 & & .394 & \\
\hline r13 & $\begin{array}{l}\text { La vacuna está en la } \\
\text { naturaleza }\end{array}$ & 4.19 & 1.80 & .764 & & .372 & \\
\hline r14 & $\begin{array}{l}\text { Las mascarillas saturan } \\
\text { el medio ambiente } \\
\text { Intencional }\end{array}$ & 4.03 & 1.25 & $\begin{array}{l}.768 \\
.783\end{array}$ & & .367 & \\
\hline r15 & $\begin{array}{l}\text { Ecologistas serían } \\
\text { relevantes en este } \\
\text { confinamiento }\end{array}$ & 4.17 & 1.54 & .767 & & & .360 \\
\hline r16 & $\begin{array}{l}\text { Ambientalistas } \\
\text { aportarían en esta } \\
\text { cuarentena }\end{array}$ & 4.15 & 1,61 & .770 & & & .386 \\
\hline r17 & $\begin{array}{l}\text { Recicladores ayudarían } \\
\text { en este encierro }\end{array}$ & 4.15 & 1.17 & .762 & & & .369 \\
\hline r18 & $\begin{array}{l}\text { Ahorradores emergerían } \\
\text { en esta crisis sanitaria }\end{array}$ & 4.10 & 1.82 & .751 & & & .375 \\
\hline r19 & $\begin{array}{l}\text { Bicitaxis serían } \\
\text { esenciales en esta } \\
\text { pandemia }\end{array}$ & 4.03 & 1.51 & .751 & & & .395 \\
\hline r20 & $\begin{array}{l}\text { Ecotaxis serían } \\
\text { indispensables en esta } \\
\text { contingencia }\end{array}$ & 4.07 & 1.63 & .703 & & & .394 \\
\hline r21 & $\begin{array}{l}\text { Mascarillas } \\
\text { contaminarían en esta } \\
\text { epidemia }\end{array}$ & 4.11 & 1.50 & .732 & & & .393 \\
\hline
\end{tabular}

Nota: Elaborada con los datos del estudio. Método: Ejes principales, Rotación: Promax. Emociones (16\% de la varianza total explicada y alfa de ,780), Cognitivas (12\% de la varianza total explicada y alfa de ,765), Intenciones (7\% de la varianza total explicada y alfa de ,753). Todos los ítems se responden con alguna de cinco opciones que van desde $0=$ "nada de acuerdo" hasta 5 = "bastante de acuerdo". 
Tabla 2. Relaciones entre variables

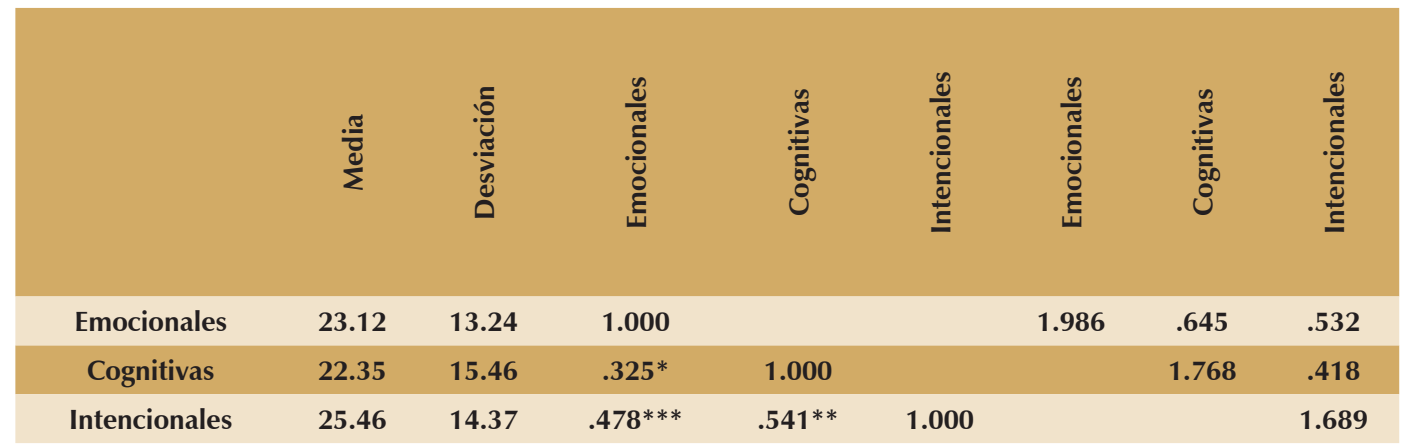

Nota: Elaborada con los datos del estudio; ${ }^{*} p<, 01 ;{ }^{* *} p<<, 001 ;{ }^{* * *} p<, 0001$

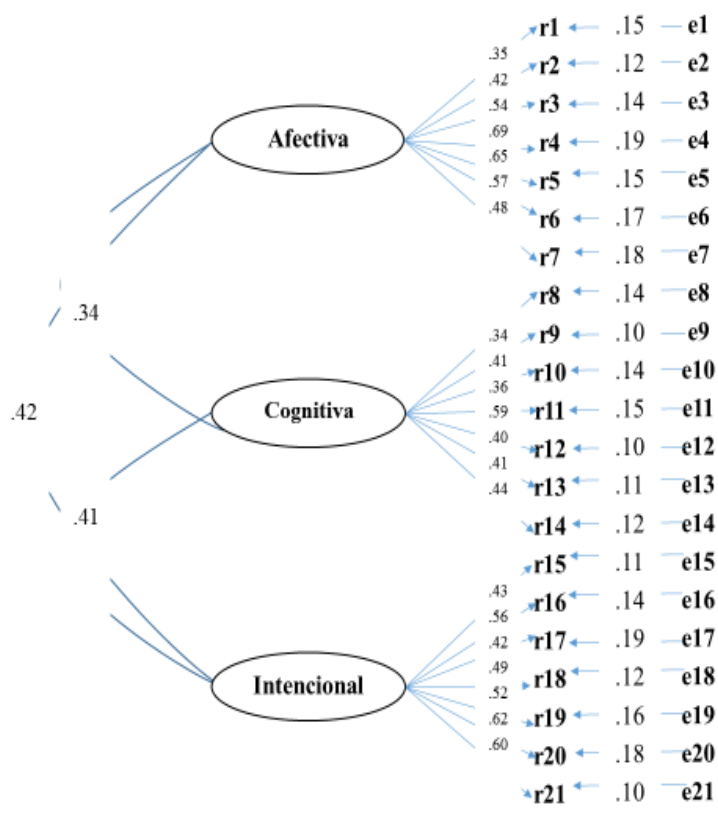

Figura 1. Estructura factorial reflejante de las actitudes

Nota: Elaborada con los datos del estudio; F1 = Actitudes emocionales, F2 = Actitudes Cognitivas, F3 = Actitudes Intencionales; $r=$ Reactivo o indicador, $\mathrm{e}==$ Error al medir el indicador o reactivo, $\bullet$ relación entre el error y el indicador, $\rightarrow$ relación entre el factor y el indicador.

En relación con la teoría de las actitudes las cuales destacan los tres componentes emotivos, cognitivos e intencionales, el presente trabajo ha demostrado la prevalencia de esa estructura de trayectorias reflejantes en un modelo, pero el porcentaje de varianza total sugiere la inclusión de un factor ambivalente que incrementaría el poder predictivo del modelo. En el aula virtual la aplicación de este hallazgo en las secuencias didácticas permitirá establecer dinámicas orientadas hacia la ambivalencia; emotividad y razonamiento en torno a sus efectos sobre los recursos y servicios públicos.
En cuanto a los estudios de las actitudes las cuales destacan la hegemonía del componente emotivo ante eventos de riesgo, el presente trabajo ha demostrado que se trata de una dimensión múltiple en la que convergen indicadores ajustados al enojo, miedo o zozobra ante los efectos de la pandemia en la naturaleza. Estos resultados permitirán el diseño de secuencias pedagógicas relativas a la educación para la sustentabilidad, principalmente en los concerniente a los eventos de riesgo.

Los marcos teóricos, conceptuales y empíricos parecen destacar la hegemonía de las disposiciones cognitivas para explicar la emergencia de actitudes ante eventos de riesgo como una pandemia. En ese sentido, la presente investigación ha destacado la estructura factorial que explica las relaciones entre las dimensiones afectiva, cognitiva e intencional como un proceso formativo de disposiciones en contra o a favor de los efectos del coronavirus en la naturaleza.

Juárez (2020) encontró tres subdimensiones cognitivos relativas a la inconmensurabilidad, impredecibilidad y controlabilidad de los efectos de la Covid-19. En este sentido, el presente trabajo demostró que esa estructura cognitiva estaría conformada por siete indicadores que miden las evaluaciones de los encuestados con respecto a sus expectativas de en una situación de crisis sanitaria prolongada. Líneas de estudio concernientes a la observación sistemática de la estructura cognitiva permitirán anticipar la organización de sectores públicos y privados, así como actores sociales y políticos.

Limón (2020) también observó la estructura cognitiva y sus indicadores referentes a las necesidades de información respecto a la Covid-19 y su relación con la naturaleza. Encontró que la muestra encuestada atendía los mensajes para comparar información siempre y cuando la fuente tuviera un prestigio bajo, o bien, los contenidos fuesen inverosímiles. En el presente trabajo se ha demostrado que esa estructura cognitiva refleja más bien contenidos ambivalentes como la generación de residuos sanitarios 
frente a la reducción de emisiones de bióxido de carbono en la atmósfera por el confinamiento de personas. Futuras áreas de estudio relativas a la estructura ambivalente permitirán anticipar conflictos entre gobernantes y gobernados ante la reducción de emisiones de $\mathrm{CO} 2$ y el aumento de basura sanitaria.

Molina (2020) ponderó la movilidad de las personas a partir del confinamiento prolongado de un mes y el desconfinamiento inmediato de las personas, encontrando efectos en la reducción del uso del transporte público, pero el incremento del aislamiento. En el presente trabajo se ha establecido un factor intencional que refleja ambivalencia al usar transporte contaminante, pero que los usuarios consideran seguro para evitar aglomeraciones. Estudios asociados a la saturación de personas en el transporte público explicarán los efectos de las políticas de escalamiento de horarios laborales.

Pérez (2020) observó diferencias significativas entre las audiencias de los medios tradicionales; televisión, radio y prensa con respecto a la agenda establecida en las redes sociales como Facebook, Twitter, Instagram, YouTube, WhatsApp, Tik Tok y Periscope. Las primeras audiencias centraban su interés en los contenidos dedicados a las visitas de especies en el confinamiento, pero las redes anunciaban el maltrato de los animales domésticos. En el presente trabajo se observó esa misma ambivalencia para la información diseminada en los medios tradicionales y las redes sociales respecto al transporte público como foco de infección. Investigaciones posteriores deberán esclarecer el impacto de las políticas de comunicación social sobre las actitudes de los ciudadanos.

En resumen, los estudios de los efectos de la Covid-19 sobre la naturaleza dan cuenta de una ambivalencia derivada de los efectos negativos y positivos de la crisis sanitaria en la naturaleza, pero la estructura factorial observada en el presente trabajo sugiere que esas actitudes reflejan sólo una parte de esa ambivalencia. Líneas de indagación futuras permitirán advertir un cuarto componente que la literatura enuncia como disposiciones ambivalentes para explicar el repudio y el apoyo de la ciudadanía para con sus gobernantes. Tal cuestión parece intensificarse en la medida en que la pandemia se prolonga.

\section{CONCLUSIÓN}

El aporte del presente trabajo al estado del conocimiento radica en la especificación de un modelo para el estudio de las actitudes hacia los efectos pandémicos en la naturaleza, aunque el diseño de la investigación limitó los resultados al escenario del trabajo, sugiriendo la extensión de este a un contexto de eventos de riesgo.
En lo consultivo, la investigación aporta los elementos centrales para la edificación de una política ambiental centrada en la cognición de las disposiciones. Se trata del diseño de secuencias pedagógicas orientadas hacia la sustentabilidad local en el manejo de los recursos y servicios municipales. Es decir, a partir de la edificación de una agenda pública será posible discernir entre los razonamientos deliberados, planificados y sistemáticos emergentes de la pandemia, el confinamiento y las crisis sanitaria y económica.

\section{REFERENCIAS}

Anguiano, F. (2020). Meta-analysis of the effects of the entrepreneurship on local development: Implications for the COVID-19 coronavirus pandemic. International Journal of Research in Engineering \& Science, 8 (9), 40-47

Bustos, J. M. (2020). Attitudes towards the effects of Covid-19 on the environment. Journal of Clinical Cas Report \& Studies, 1 (3), 1-5

Carreón, J. (2020). Coffee entrepreneurship during Covid-19. Revista Especializada en Tecnología e Ingeniería, 14 (1), $11-20$

Hernández, J. (2020). Specification of a social intervention model against COVID-19. Biomedical of Scientific \& technical Research, 26 83), 62-65

Juárez, M. (2020). La percepción de riesgo en estudiantes universitarios ante la propagación del coronavirus SARSCoV-2 y la enfermedad Covid-19. Revista de Psicología, $8(17), 94-107$

Limón, G. A. (2020). Specification of a model of need for information about COVID-19 coronavirus. International Journal of Social science Studies, 8 (6), 1-5

Molina, H. D. (2020). Una aproximación estadística al comportamiento de brote Covid-19 en la China Continental. Tepexi, 7 (14), 6-16

Organización Mundial de la Salud (2020). Brote de enfermedad por coronavirus (Covid-19). New York OMS https://www.paho.org/es/temas/coronavirus/broteenfermedad-por-coronavirus-covid-19

Pérez, G. (2020). Attitudes toward the media spread of the COVID-19 coronavirus. International Journal of Humanities \& Social Science Invention, 9 (8), 20-25 